\title{
Correction: Discontinuation of adalimumab after achieving remission in patients with established rheumatoid arthritis: 1-year outcome of the HONOR study
}

Tanaka Y, Hirata S, Kubo S, et al. Discontinuation of adalimumab after achieving remission in patients with established rheumatoid arthritis: 1-year outcome of the HONOR study. Ann Rheum Dis 2015;74:389-95. In table 3 (B) values of 95\% CI were not correct. The corrected table $3(\mathrm{~B})$ is given below.

\begin{tabular}{lllll}
\hline \multicolumn{7}{l}{ Table 3 (B) Multivariate logistic regression analysis } & \\
\hline Items & Odds & $95 \% \mathrm{Cl}$ & Chi-square & $\mathrm{p}$ Value \\
Age (years) & 0.963 & 0.906 to 1.024 & 1.441 & 0.2300 \\
ADA admin periods (weeks) & 0.985 & 0.959 to 1.011 & 1.254 & 0.2629 \\
DAS28-ESR & 0.143 & 0.029 to 0.711 & 5.653 & $0.0174^{*}$ \\
RF (U/mL) & 1.012 & 0.996 to 1.028 & 2.127 & 0.1448
\end{tabular}

Univariate logistic regression analysis was performed using items with $p<0.1$ in table 2 to investigate factors related to sustained remission for 1 year after ADA discontinuation. Then, multivariate analyses were conducted using the variables with $\mathrm{p}<0.2$ in the univariate analysis. Using the DAS28-ESR values which were significant in logistic analysis, ROC analysis was conducted with the response (dependent) variable of if DAS28-ESR $<2.6$ (1) or $\geq 2.6$ (0) 1 year after discontinuation of ADA and the explanatory variable of DAS28-ESR at the timing of ADA discontinuation.

${ }^{*} p<0.01$ : Wald test.

ADA, adalimumab; DAS28, disease activity score 28; RF, rheumatoid factor. 